\section{Exceptional responders: in search of the science behind the miracle cancer cures}

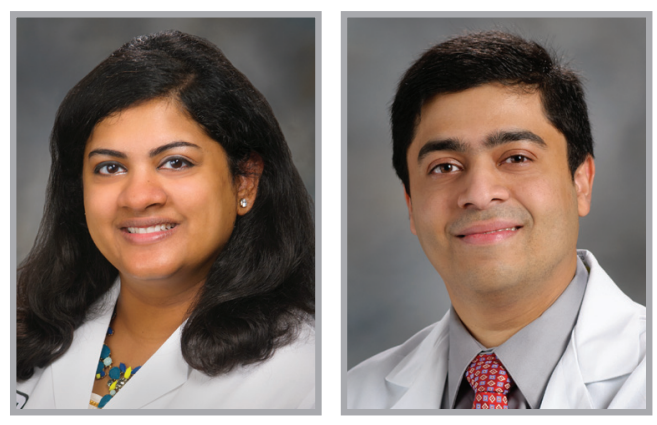

“The physician must be able to tell the antecedents, know the present, and foretell the future."

Ishwaria Mohan Subbiah¹ \& Vivek Subbiah ${ }^{* 2,3}$

"In solving a problem of this sort, the grand thing is to be able to reason backward. That is a very useful accomplishment, and a very easy one, but people do not practice it much. In the everyday affairs of life it is more useful to reason forward, and so the other comes to be neglected."

- Sherlock Homes in A Study in Scarlet (Sir Arthur Conan Doyle)

The individual and societal impact of cancer remains a problem of Herculean proportions and one that is expected to outpace cardiovascular disease as the primary killer of humans in developed nations. With such a rise in incidence comes the need for the rapid development of novel therapies. The advent of next-generation sequencing technologies has led to a rapid expansion of the molecular profiling of patients with cancer. Such modalities are not only available at most large institutions and comprehensive cancer centers; they are also freely accessible commercially through various companies to the practicing oncologist in the community. This newfound knowledge of somatic mutations within a patient's molecular profile, as well as a rise in the fundamental understanding of the cellular processes behind cancer cell deregulation, have spurred the rapid growth of the development of novel targeted therapies.

The impetus towards patient benefit has driven countless new drugs through the preclinical development phase and into Phase I clinical trials. This need for novel therapies and the rapid development of new drugs has been further supported by the regulatory authorities. In response to the growing backlash against the laboriously slow drug development process, the US FDA introduced varying tracks of drug approval in which the most promising agents with remarkable responses in earlyphase clinical trials have been fast tracked for approval with postmarketing requirements in order to confirm their long-term benefit and safety [1].

\section{KEYWORDS}

- exceptional responder • genomics

- next-generation sequencing

- outlier responder • unusual responder

\author{
“The advent of next-generation \\ sequencing technologies has led \\ to a rapid expansion of the \\ molecular profiling of patients \\ with cancer.”
}


"There has to be a real-time, open access online registry that stores the data relating to all of these 'miracle' patients and all of the data that has been deposited so that all of this investigative work is accessible and useful for the oncology community at large."

\section{"The grand thing is to be able to reason backward" - a Sherlock Holmesian approach}

Certainly, not every new drug tested with a novel mechanism of action or a novel formulation has led to remarkable patient responses. Indeed, Phase I trials have quickly recognized not only toxicities and pharmacokinetic and pharmacodynamic properties of the $\operatorname{drug}(s)$ under investigation, but also tumor responses (or a lack thereof), particularly through expansion cohorts at the maximum tolerated dose and the recommended Phase II dose. The practicing oncologist and clinical investigator catch a glimpse of these novel drugs' Phase I results at the annual oncology conferences. At these conferences, we pay brief but necessary heed to the safety and tolerance of the drug (e.g., the number of grade 3 or 4 toxicities), but our attention quickly turns towards the end of the poster where, if one is fortunate, the trial investigators report the patient's tumor responses on the study. With this passing glance at a tumor response rate, we tend to dismiss the drug from further development when the tumor response rate is reported as $5 \%$. However, upon closer inspection, it may become evident that one patient attained a complete response, showing no radiographic evidence of the cancer where previously widely metastatic cancer was noted. These are the 'exceptional responders' [2,3]. Their story is one that is repeated through select Phase I and even Phase II clinical trials, where a select few attain remarkable control of their disease, with some fortunate enough to experience durable responses, while other responses are more transient. These exceptional responders are often heavily pretreated through multiple lines of standard-of-care therapies and were subsequently referred for Phase I trials with hopeful intentions [3]. Scientists and physicians are detectives at heart [4]. The in-depth analysis of these n-of- 1 outlier responders calls for an approach worthy of Sherlock Holmes, where "the grand thing [is to be able] to reason backward" with the hope of unraveling unique insights into the disease that may help the current patient and future patients with the same disease or same aberration.

We will discuss a few exemplar illustrative cases that would be conventionally regarded as statistical outliers. These can give a glimpse into potentially unravelling the basis of response, even in heavily pretreated patients.
Exceptional responder leads to a

\section{clinical trial}

One such patient is a 26-year-old woman with Hodgkin's lymphoma who presented after relapsing following nine standard lines of therapy, including an allogeneic stem cell transplant. She was initiated on a mTOR and a histone deacetylase inhibitor therapy, leading to a nearcomplete response and enabling her to receive a second transplant [5]. Immunocorrelative and proteomic analysis revealed new insights into the biology of Hodgkin's lymphoma in terms of the response signature that centered on an immune dysregulation involving an imbalance between effector and functional Tregs, in addition to targeting the mTOR pathway [5]. This case study highlights the need for an approach that focuses on combinatorial therapies that target both the pathogenesis and adaptive responses to envisioned therapies. Based on this single patient response, a Phase I clinical trial with combinatorial vorinostat and sirolimus was amended to include Hodgkin's lymphoma patients, and the preliminary results demonstrate a breakthrough for advanced Hodgkin's lymphoma.

\section{Deep sequencing uncovers unique aberrations}

In another in-depth analysis of a metastatic bladder cancer patient with an exceptional response to everolimus, whole-genome sequencing identified a loss-of-function mutation in TSC1, a regulator of the mTOR pathway activation, as the basis of response [6]. A second patient with a difficult-to-describe malignant neoplasm that defied conventional histologic and immunohistochemical characterization presented a diagnostic and therapeutic challenge. This patient, with an obscure sarcoma-like undifferentiated lesion that failed conventional chemotherapies, responded in a Phase I trial combining sorafenib, temsirolimus and bevazicumab [7]. Clinical next-generation sequencing identified a KIAA1549-BRAF fusion and PTEN loss in this patient, identifying the basis of response to be a dual inhibition of RAF kinase and mTOR [7]. This case was the first report of this sarcomatype tumor driven by a KIAA1549-BRAF fusion responding to sorafenib-based combination therapy [7]. In another exceptional responder study, whole-exome sequencing of a urothelial carcinoma patient with a complete response lasting 14 months in a Phase I trial of pazopanib and everolimus revealed two concurrent mutations 
in mTOR, the target of everolimus, namely an activating mutation $\left(m T O R^{E 2419 K}\right)$ and a second mutation $\left(m T O R^{E 2014 K}\right)$, which were also confirmed by preclinical validation studies [8]. Even in aggressive malignancies, such as small-cell lung cancer, whole-genome sequencing of exceptional responders to combined CHK1 inhibition and DNA-damaging chemotherapy [9] revealed a clonal hemizygous mutation in the Mre11 complex gene $R A D 50$ that attenuated ataxia telangiectasia mutated-dependent signaling, which, in the context of CHK1 inhibition, contributed to the extreme sensitivity to irinotecan via synthetic lethality [9].

\section{'Positive' responders in 'negative' clinical trials}

There were over 25 IGF1R inhibitor-related molecules in preclinical or clinical development in the last decade. However, the continued development of these agents has stalled, in part due to aggregate negative trials. Nevertheless, there have been several outlier responder patients, especially in Ewing's sarcoma, an orphan disease that responds dramatically to IGF1R-targeted therapies. In a pilot study, two patients with Ewing's sarcoma demonstrated remarkable responses to single-agent IGF1R therapy and then relapsed [10]. Clinically, both patients reresponded to a combination of an IGF1R and mTOR inhibitor therapy. Phosphorylated (p)AKT and p-mTOR upregulation occurred in the resistant tissue, demonstrating the mTOR pathway to be the mechanism of acquired resistance to single-agent IGF1R inhibitor therapy [10]. One patient continued to respond while the second patient progressed - their resistant tissue revealed concurrent activation of the ERK pathway as a potential mechanism of resistance. Therefore, unusual responders represent a moving, dynamic target requiring rapid study of their tumor tissue, virtually in real-time. Further deep sequencing identified mutations in PTPRD and GRB10 in the patient with Ewing's sarcoma achieving complete response [11]. Moreover, the PTPRD mutation was determined to be a germline mutant. The analysis of additional patients with Ewing's sarcoma revealed a novel germline mutation in the PTPRD gene in three out of eight patients (37.5\%) with metastatic Ewing's sarcoma [12]. Such studies of outlier patients have yielded several insights into the pathogenesis of disease.

Although the preclinical success of the recombinant human protein Apo2L/TRAIL (dulanermin) did not translate into the anticipated success in clinical trials and the clinical development stalled, there have been exceptional responders to this agent. In one such study, a patient with refractory chondrosarcoma had a prolonged response to dulanermin. DR4 was detected in the patient's tumor, which may have enabled this response. A resistant tumor tissue sample showed upregulation of prosurvival proteins, namely p-NF- $\kappa$ Bp65 (Ser-536), p-STAT3 (Tyr-705), p-ERK 1/2 (Thr-202/Tyr204), p-mTOR (Ser-2448), FASN and Bcl-2, which were detected after the patient developed acquired resistance upon therapy [13]. Analyses of every resistant sample may enable adaptive responses to future combinatorial therapy.

There have been three separate trials of sunitinib (a VEGFR2/PDGFR- $\beta$ /c-kit/FLT3/ RET/CSF1R inhibitor) in germ cell tumors assessing 33, ten and five patients, respectively [14-16]. Although the trials as an aggregate were negative, sunitinib conferred clinical benefit in some patients. In the trial that enrolled only five patients, sunitinib conferred clinical benefit to a heavily pretreated patient [16]. Next-generation sequencing of this exceptional responder identified the first reported case of a RET amplification as a potential basis for the sensitivity to sunitinib in this patient with a refractory germ cell tumor [16].

Whereas such cases would once have been buried in the depths of the overlooked manuscripts detailing their 'negative trials', major forces in cancer care are now taking notice. The National Cancer Institute has organized a multiinstitutional effort bringing together prominent cancer programs throughout the USA, in addition to special programs in major cancer centers $[2,17]$. Through multilevel pan-omics profiling, the technology is now in place to decipher the mechanisms of response. Given that such extreme responder cases are exponentially increasing, how should scientists and physicians keep track of them? There has to be a real-time, open access online registry that stores the data relating to all of these 'miracle' patients and all of the data that has been deposited so that all of this investigative work is accessible and useful for the oncology community at large.

As Hippocrates aptly put it, "The physician must be able to tell the antecedents, know the present, and foretell the future." Studying exceptional responders may be one such way of achieving this.
“There has to be a realtime, open access online registry that stores the data relating to all of these 'miracle' patients and all of the data that has been deposited so that all of this investigative work is accessible and useful for the oncology community at large." 
Financial \& competing interests disclosure The University of Texas MD Anderson Cancer Center is supported in part by a Cancer Center Support Grant (CA016672) from the NIH. The authors have no other relevant affiliations or financial involvement with any organization or entity with a financial interest in or financial conflict with the subject matter or materials discussed in the manuscript apart from those disclosed.

No writing assistance was utilized in the production of this manuscript.

\section{References}

1 Yao JC, Meric-Bernstam F, Lee JJ, Eckhardt SG. Accelerated approval and breakthrough therapy designation: oncology drug development on speed? Clin. Cancer Res. 19(16), 4305-4308 (2013).

2 Abrams J, Conley B, Mooney M et al. National Cancer Institute's Precision Medicine Initiatives for the new National Clinical Trials Network. Am. Soc. Clin. Oncol. Educ. Book 2014, 71-76 (2014).

3 Mullard A. Learning from exceptional drug responders. Nat. Rev. Drug Discov. 13(6), 401-402 (2014).

4 Brannon AR, Sawyers CL. ' $N$ of 1' case reports in the era of whole-genome sequencing. J. Clin. Invest. 123(11), 4568-4570 (2013).

5 Subbiah V, Brown RE, McGuire MF et al. A novel immunomodulatory molecularly targeted strategy for refractory Hodgkin's lymphoma. Oncotarget 5(1), 95-102 (2014).

6 Iyer G, Hanrahan AJ, Milowsky MI et al. Genome sequencing identifies a basis for everolimus sensitivity. Science 338(6104), 221 (2012).

7 Subbiah V, Westin SN, Wang K et al. Targeted therapy by combined inhibition of the RAF and mTOR kinases in malignant spindle cell neoplasm harboring the KIAA1549-BRAF fusion protein. J. Hematol. Oncol. 7(1), 8 (2014)

8 Wagle N, Grabiner BC, Van Allen EM et al. Activating mTOR mutations in a patient with an extraordinary response on a Phase I trial of everolimus and pazopanib. Cancer Discov. 4(5), 546-553 (2014).

9 Al-Ahmadie H, Iyer G, Hohl M et al. Synthetic lethality in ATM-deficient RAD50-mutant tumors underlie outlier response to cancer therapy. Cancer Discov. 4(9), 1014-1021 (2014).

10 Subbiah V, Naing A, Brown RE et al. Targeted morphoproteomic profiling of Ewing's sarcoma treated with insulin-like growth factor 1 receptor (IGF1R) inhibitors: response/resistance signatures. PLoS ONE 6(4), e18424 (2011).

11 Jiang Y, Subbiah V, Janku F et al. Novel secondary somatic mutations in Ewing's sarcoma and desmoplastic small round cell tumors. PLoS ONE 9(8), e93676 (2014).

12 Jiang Y, Janku F, Subbiah V et al. Germline PTPRD mutations in Ewing sarcoma: biologic and clinical implications. Oncotarget 4(6), 884-889 (2013).
13 Subbiah V, Brown RE, Buryanek J et al. Targeting the apoptotic pathway in chondrosarcoma using recombinant human Apo2L/TRAIL (dulanermin), a dual proapoptotic receptor (DR4/DR5) agonist. Mol. Cancer Ther. 11(11), 2541-2546 (2012).

14 Oechsle K, Honecker F, Cheng T et al. Preclinical and clinical activity of sunitinib in patients with cisplatin-refractory or multiply relapsed germ cell tumors: a Canadian Urologic Oncology Group/German Testicular Cancer Study Group cooperative study. Ann. Oncol. 22(12), 2654-2660 (2011).

15 Feldman DR, Turkula S, Ginsberg MS et al. Phase II trial of sunitinib in patients with relapsed or refractory germ cell tumors. Invest. New Drugs 28(4), 523-528 (2010).

16 Subbiah V, Meric-Bernstam F, Mills GB et al. Next generation sequencing analysis of platinum refractory advanced germ cell tumor sensitive to sunitinib (Sutent ${ }^{\circledR}$ ) a VEGFR2/ PDGFRss/c-kit/ FLT3/RET/CSF1R inhibitor in a Phase II trial. J. Hematol. Oncol. 7(1), 52 (2014).

17 Sheridan C. Cancer centers zero in on exceptional responders. Nat. Biotechnol. 32(8), 703-704 (2014). 